\title{
A Situação Brasileira do Atendimento Pedagógico-Educacional Hospitalar
}

Eneida Simões da Fonseca

Universidade do Estado do Rio de Janeiro

\section{Resumo}

A legislação brasileira reconhece o direito de crianças e jovens hospitalizados (CNDCA, 1995) ao atendimento pedagógico-educacional, durante seu período de internação. Esta modalidade de atendimento denomina-se classe hospitalar, segundo terminologia do MEC/SEESP (1994). A inexistência de teorias ou estudos desta natureza em território nacional gera, tanto na área educacional quanto na de saúde, o desconhecimento desta modalidade de ensino e integralização da atenção de saúde às crianças e aos jovens hospitalizados. Considerando este fato, o presente estudo realizou um levantamento nacional dos estados federativos que oferecem o atendimento de classe hospitalar e as formas como este é ministrado.

No Brasil, há 39 classes hospitalares distribuídas e em funcionamento em 13 unidades federadas. Esse tipo de atendimento decorre, em sua maioria, de convênio firmado entre as Secretarias de Educação e de Saúde dos estados, embora existam classes hospitalares resultantes de iniciativas de entidades filantrópicas e universidades. Noventa e cinco professores atuam nessa modalidade de ensino, atendendendo mais de 2.000 crianças/mês na faixa etária entre 0 e 15 anos de idade. Abre-se, com este estudo, a necessidade de formular propostas e aprofundar conhecimentos teóricos e metodológicos, com vistas a, efetivamente, atingir o objetivo de dar continuidade aos processos de desenvolvimento psíquico e cognitivo das crianças e jovens hospitalizados. Faz-se necessária a elaboração de uma política voltada para as necessidades pedagógico-educacionais e os direitos à educação e à saúde desta clientela em particular etapa de vida quanto ao crescimento e desenvolvimento físico e emocional.

\section{Palavras-chaves}

Classe Hospitalar - Atenção à Diversidade - Educação Especial.
Correspondência para: Rua São Francisco Xavier, 643 20550-011 - Maracanã - RJ email: eneida@uerj.br 


\section{Current Situation Of The Pedagogical-Educational Service In Brazilian Hospitals}

Eneida Simões da Fonseca

Universidade do Estado do Rio de Janeiro

\begin{abstract}
The Brazilian legislation recognizes the right of children and youngsters to pedagogical-educational service during their stay in a hospital (CNDCA, 1995). This type of service is called hospital class according to MEC/SEESP (1994) terminology. The absence of theories or studies about this service in Brazil contributes to making this form of teaching and broad health care little known both to the educational and to the health sectors. Taking this fact into account, the present study carried out a nationwide survey of states that actually offer hospital classes and how they are given.

There are 39 hospital classes functioning in Brazil distributed among 12 States and the Federal District. This type of service is usually offered through agreements celebrated between the corresponding education and health state secretariats, although there are cases of hospital classes maintained by philanthropic groups and universities. Ninety-five teachers work in these services assisting more than 2000 children per month from 0 to 15 years of age.

Let us raise with this study the need to make proposals and expand theoretical and methodological knowledge with a view to positively reach the goal of continuing with the psychic and cognitive development of children and youngsters in hospitals. It is necessary to define policies that fulfill the pedagogical-educational needs as well as the right to education and health of this clientele that find themselves at a special moment of their physical and emotional development.
\end{abstract}

\section{Key words}

Hospital Class - Attention To Diversity - Special Education.
Correspondence:

Rua São Francisco Xavier, 643

20550-011 - Maracanã - RJ

email: eneida@uerj.br 


\section{Introdução}

A legislação brasileira reconhece o direito de crianças e jovens hospitalizados ao atendimento pedagógico-educacional. A Declaração dos Direitos da Criança e do Adolescente Hospitalizados decorreu de formulação da Sociedade Brasileira de Pediatria e da Resolução do Conselho Nacional dos Direitos da Criança e do Adolescente, com a chancela do Ministério da Justiça em 1995. Essa modalidade de atendimento denomina-se classe hospitalar, prevista pelo Ministério da Educação e do Desporto em 1994, por meio da publicação da Política Nacional de Educação Especial (MEC/SEESP, 1994). A insuficiência de teorias e estudos desta natureza em território brasileiro gera, tanto na área educacional, quanto na área de saúde, o desconhecimento desta modalidade de atendimento tanto para viabilizar a continuidade da escolaridade àquelas crianças e adolescentes que requerem internação hospitalar, quanto para integralizar a atenção de saúde e potencializar o tratamento e o cuidado prestados à criança e ao adolescente.

Abre-se, com este estudo, a necessidade de formular propostas e aprofundar conhecimentos teóricos e metodológicos, com vistas a, efetivamente, atingir o objetivo de dar continuidade ao processo de desenvolvimento psíquico e cognitivo de crianças e jovens hospitalizados, e à elaboração de uma política voltada para as necessidades pedagógico-educacionais e os direitos à educação e à saúde desta clientela que encontra-se em particular etapa de vida, tanto em relação ao crescimento e desenvolvimento, quanto em relação à construção de estratégias sócio-interativas para o viver individual e em coletividade.

\section{Objetivos}

0 presente estudo teve como objetivo básico realizar um levantamento da existência de classes hospitalares nos diversos Estados do país, buscando detectar seus elementos estru- turais na organização da atenção integral $\dot{a}$ saúde da criança e do adolescente, e na organização de oferta educacional especial à criança e ao adolescente hospitalizados. Buscou-se obter informações sobre:

- quais hospitais abrigam classes hospitalares;

- quantos professores atuam em classe hospitalar e qual sua formação;

- quantos alunos, em média, são atendidos por mês, sua faixa etária, problemática de saúde e escolaridade;

- que política e/ou diretrizes norteiam o trabalho.

\section{Metodologia}

A pesquisa aqui apresentada é parte integrante do Programa de Pesquisas para o Desenvolvimento de Estratégias Ligadas aos Direitos e Necessidades Pedagógico-Educacionais de Crianças e Jovens Hospitalizados (Fonseca, 1995b).

Inicialmente, por meio de contato com o Ministério da Educação e do Desporto (Secretaria de Educação Especial), obteve-se a listagem de endereços das Secretarias de Educação/Educação Especial dos estados brasileiros e do Distrito Federal. A pesquisa dividiu-se em duas etapas subseqüentes: na primeira, realizou-se o envio de correspondência, via correio, a todas as Secretarias de Educação/Educação Especial das 27 unidades federadas do país (26 estados e o Distrito Federal). Tal correspondência continha uma carta-resumo com os objetivos da pesquisa. Constava também um questionário de múltipla escolha com oito blocos de questões e um quadro para preenchimento dos dados de funcionamento das classes hospitalares. Todos estes documentos foram encaminhados, conjuntamente, com uma carta contendo o prazo de devolução e o endereço para envio da resposta.

Uma semana após o vencimento do prazo de devolução, os Estados que ainda não 
haviam enviado sua resposta foram contatados, via telefone, e outra cópia do questionário enviada em caso de extravio da correspondência inicial. As Secretarias que já haviam remetido sua resposta receberam carta de agradecimento da coordenação da pesquisa.

Na segunda etapa da pesquisa, cada classe hospitalar, conhecida pelas informações das Secretarias de Educação/Educação Especial durante a primeira etapa, recebeu correspondência contendo uma carta-convite, explicando os objetivos da pesquisa e prazos para a devolução das novas informações. Foram enviados um questionário e um quadro para detalhamento da realidade da classe hospitalar. Não foi previsto, nesta fase, o contato telefônico posterior ao envio das correspondências. Em ambas as etapas, foi assegurado à Secretaria de Educação/Educação Especial ou ao hospital informante que, ao final da análise dos dados, receberiam um resumo dos resultados da pesquisa.

A coleta de dados ocorreu entre os meses de julho de 1997 e fevereiro de 1998.

Os dados foram analisados descritivamente e traduzidos em percentuais quando necessário a uma melhor compreensão dos resultados.

\section{Resultados}

Das 27 correspondências inicialmente enviadas, obteve-se um retorno final de 23 delas (85,2\%). Apenas quatro Estados (14,8\%) deixaram de responder ao questionário e/ou não puderam ser contatados após envio da correspondência inicial. A tabela abaixo resume o processo de coleta de dados.

\begin{tabular}{||l|c|c|}
\hline \multicolumn{1}{|c|}{ Procedimento } & $\begin{array}{c}\mathbf{N}^{\mathbf{0}} \text { de } \\
\text { Estados* }^{*}\end{array}$ & $\mathbf{\%}$ \\
\hline Resposta após carta & 12 & 44 \\
\hline Resposta após telefonema & 11 & 41 \\
\hline Impossibilidade de contato & 4 & 15 \\
\hline Total & $\mathbf{2 7}$ & $\mathbf{1 0 0}$ \\
\hline
\end{tabular}

*Inclusive o Distrito Federal.
A pesquisa apontou um total de 11 unidades federadas (41\%) - dez Estados e o Distrito Federal - oferecendo atendimento pedagógico-educacional para crianças e adolescentes hospitalizados, isto é, dispondo de classes hospitalares, conforme terminologia do MEC/SEESP (1994).

A tabela, a seguir, mostra, por região, o número de Estados que dispõem de atendimento pedagógico-educacional para crianças hospitalizadas.

Unidades Federadas com Classes Hospitalares (CH), por Região

\begin{tabular}{|l|c|c||}
\hline \multicolumn{1}{|c|}{ Região } & $\begin{array}{c}\text { No de } \\
\text { Estados }\end{array}$ & $\begin{array}{c}\mathbf{N}^{0} \text { de Estados } \\
\text { Com CH }\end{array}$ \\
\hline Norte & 7 & 1 \\
\hline Nordeste & 9 & 3 \\
\hline Centro-Oeste & 4 & 2 \\
\hline Sudeste & 4 & 3 \\
\hline Sul & 3 & 2 \\
\hline Total & $\mathbf{2 7}$ & $\mathbf{1 1}$ \\
\hline
\end{tabular}

Não foram identificadas classes hospitalares em 12 unidades federadas (44,4\%), em 1997. Os motivos para a não oferta de classes hospitalares foram variados. Um Estado da região Sudeste $(3,7 \%)$ informou ter sido esta modalidade de atendimento descontinuada devido às mudanças na rotina de internação e tratamento médico dos hospitais daquela unidade federativa (MG).

Somaram 11 os Estados que nunca ofereceram atendimento pedagógico-educacional para crianças e jovens hospitalizados. Destes, seis Estados $(22,2 \%)$ já consideraram a possibilidade de implantar o serviço, embora não tenham chegado a implementá-lo; e os outros cinco $(18,5 \%)$ disseram nunca terem considerado tal possibilidade por desconhecerem esta modalidade de atendimento.

Dos Estados que consideraram a implantação do serviço, três deles alegaram que 
a impossibilidade para tal efetivação se devia a motivos, tais como: falta de profissional especializado para dar suporte ao trabalho, falta de professores para este tipo de atuação e falta de recursos financeiros.

Os três outros Estados alegaram não haver necessidade de classe hospitalar, por falta de demanda e/ou por nunca ter havido solicitação de familiares, comunidades ou órgãos oficiais. A tabela seguinte resume as situações de oferta da classe hospitalar, segundo a condição de planejamento atual e pregresso, nas diferentes unidades federadas (inclusive o Distrito Federal).

Oferta de Classes Hospitalares por Estado (inclusive 0 Distrito Federal)

\begin{tabular}{||l|c|c||}
\hline \multicolumn{1}{|c|}{ Situação } & $\begin{array}{c}\text { No de } \\
\text { Estados }\end{array}$ & $\mathbf{\%}$ \\
\hline Oferece atualmente & 11 & 41 \\
\hline Ofereceu no passado & 1 & 3,7 \\
\hline $\begin{array}{l}\text { Nunca ofereceu, mas já } \\
\text { considerou a possibilidade }\end{array}$ & 6 & 22,2 \\
\hline $\begin{array}{l}\text { Nunca ofereceu nem } \\
\text { considerou a possibilidade }\end{array}$ & 5 & 18,5 \\
\hline Sem informação & 4 & 14,8 \\
\hline Total & $\mathbf{2 7}$ & $\mathbf{1 0 0}$ \\
\hline
\end{tabular}

\section{A implantação do atendimento de classe hospitalar}

Das classes hospitalares que informaram o ano de início de suas atividades, situam-se na região Sudeste as duas classes com mais longo tempo de atuação; a primeira, aberta em 1950; e a segunda, em 1953. O Município do Rio de Janeiro tem a mais antiga classe hospitalar em funcionamento no país, a classe hospitalar do Hospital Municipal Jesus (hospital público infantil). Esta classe iniciou oficialmente suas atividades em 14 de agosto de 1950. A tabela, a seguir, resume o número de classes hospitalares implantadas e ainda em funcionamento.

Implantação de Classes Hospitalares

\begin{tabular}{||l|c|}
\hline \multicolumn{1}{|c|}{ Ano } & $\mathbf{N}^{\circ}$ de $\mathbf{~ H ~}$ \\
\hline até 1950 & 1 \\
\hline $1951-1960$ & 1 \\
\hline $1961-1970$ & 1 \\
\hline $1971-1980$ & 1 \\
\hline $1981-1990$ & 8 \\
\hline 1991 até dez. 1997 & 9 \\
\hline Sem informação & 9 \\
\hline Total & $\mathbf{3 0}$ \\
\hline
\end{tabular}

Conforme observado na tabela anterior, foi a partir de 1981 que o atendimento de classe hospitalar teve um aumento significativo no número de classes implantadas. 0 crescimento do número de classes hospitalares coincide com o redimensionamento do discurso social sobre a infância e a adolescência, que culminou com a aprovação do Estatuto da Criança e do Adolescente e seus desdobramentos posteriores.

\section{O hospital onde a classe hospitalar funciona}

Um total de 30 hospitais no Brasil conta com atendimento pedagógico-educacional para crianças e jovens hospitalizados.

A tabela, a seguir, resume, por região, o número de hospitais com classes hospitalares, assim como o quantitativo de classes hospitalares que responderam à segunda etapa da pesquisa. 
Classes Hospitalares por Região

\begin{tabular}{||l|c|c|c|}
\hline Regiões & $\begin{array}{c}\text { Hospi- } \\
\text { tais } \\
\text { Com CH }\end{array}$ & $\begin{array}{c}\text { CH que } \\
\text { Respondeu } \\
\text { à 2 }{ }^{\text {a }} \text { Etapa }\end{array}$ & $\%$ \\
\hline Norte & 02 & 01 & 50 \\
\hline Nordeste & 03 & 01 & 33 \\
\hline Centro-0este & 09 & 03 & 33 \\
\hline Sudeste & 10 & 06 & 60 \\
\hline Sul & 06 & 05 & 83 \\
\hline Total & $\mathbf{3 0}$ & $\mathbf{1 6}$ & $\mathbf{5 3}$ \\
\hline
\end{tabular}

A metade mais uma das classes hospitalares identificadas no país, por meio desta pesquisa, responderam à sua $2^{\text {a }}$ etapa.

Quanto ao tipo de hospital no qual as classes hospitalares funcionam, a maioria (37\%) encontra-se em hospital geral público com enfermaria pediátrica $(n=11)$. Seis classes hospitalares (20\%) funcionam em hospitais públicos infantis, sendo uma delas em hospital infantil de oncologia. Quatro classes hospitalares (13\%) funcionam em hospitais mantidos pela Santa Casa de Misericórdia. Uma outra classe hospitalar (3\%) funciona em hospital privado de oncologia. As demais oito classes hospitalares (27\%) funcionam em outros tipos de hospitais públicos, mas com especificidades em seus atendimentos, como ortopedia, oncologia, cardiologia, queimados e psiquiatria, e que oferecem enfermarias pediátricas.

\section{A rotina do hospital}

Além das atividades pedagógico-educacionais oferecidas pelas classes hospitalares, buscou-se obter dados sobre as visitas permitidas às crianças, presença dos pais ou outro adulto durante a internação, e oferta de serviço de recreação (eventual e/ou permanente).

Todas as crianças e jovens internados nos hospitais constantes deste estudo dispõem de acompanhamento por familiar ou adulto, durante todo o período de hospitalização.
Cinqüenta por cento dos hospitais oferecem aos familiares e amigos das crianças ou adolescentes hospitalizados um mínimo de uma hora diária para visitas. Nestes hospitais, o horário de visitas ocorre entre as $15 \mathrm{e}$ as 18 horas.

Em 44\% dos hospitais que responderam à segunda etapa da pesquisa $(n=7)$, as crianças dispõem de serviço de recreação oferecido por profissionais lotados nesses hospitais. A presença dos Doutores da Alegria nas enfermarias foi mencionada por seis dos hospitais participantes da segunda etapa do presente estudo. Para estes hospitais, a proximidade entre os referenciais teóricos, ao dispor do atendimento pedagógico-educacional, e do serviço de recreação não implicou a sua fusão, preservando estruturas distintas de atenção. A educação lúdica ou o brincar como promoção de saúde não substituíram a necessidade de as classes hospitalares se ocuparem com as questões didático-pedagógicas da produção de conhecimento e da produção de relações de aprendizagem.

\section{O ambiente físico da classe hospitalar}

Os dados aqui apresentados referem-se apenas às classes hospitalares participantes da segunda etapa da pesquisa $(n=16)$, uma vez que a primeira etapa não incluiu informações sobre o ambiente físico e específico de cada classe hospitalar.

0 atendimento pedagógico-educacional oferecido por estas classes hospitalares é, em $62,5 \%$ delas $(n=10)$, desenvolvido em salas exclusivas cedidas pelo hospital e/ou salas adaptadas ou utilizadas no horário das aulas (escola hospitalar). Destas, três classes hospitalares contam, também, com sala de secretaria, e duas outras possuem sala para depósito de materiais diversos que são utilizados pelos professores. As demais seis classes hospitalares $(37,5 \%)$ têm suas atividades pedagógico-educacionais realizadas exclusivamente nas próprias enfermarias (junto aos leitos das crianças e adolescentes). 
A tabela seguinte resume os dados do ambiente físico disponível às classes hospitalares.

Ambiente Físico das Classes Hospitalares

\begin{tabular}{||l|c||}
\hline \multicolumn{1}{|c|}{ Espaço Físico Disponível } & No $^{|c| \mid}$ \\
\hline \multicolumn{2}{|c|}{ Escola Hospitalar } \\
Salas de Aula & 05 \\
\hline Salas de Aula + Secretaria & 03 \\
\hline Salas de Aula + Secretaria + Depósito & 02 \\
\hline Subtotal & 10 \\
\hline Atendimento nas Enfermarias & 06 \\
\hline Sem Informação & 14 \\
\hline Total & 30 \\
\hline
\end{tabular}

A disponibilidade de espaços próprios (escola hospitalar) para o atendimento pedagógico-educacional não invalida o atendimento junto ao leito para as crianças que assim o necessitarem (crianças em isolamento imunoprotetor, crianças em unidades de intensivismo pediátrico etc.)

Quanto aos turnos de atendimento pedagógico-educacional no ambiente hospitalar, $44 \%$ das classes hospitalares $(n=7)$ dispõem de professores que atuam no horário da manhã e professores que atuam no turno da tarde, 33\% das classes hospitalares $(n=5)$ funcionam exclusivamente no horário da tarde (entre $12 \mathrm{~h} 30$ e 18 horas) e $25 \%$ das classes hospitalares $(n=4)$ contam com professores que atuam em horário integral (manhã e tarde).

\section{Perfil docente}

A presente pesquisa mapeou um total de 80 professores exercendo atividades pedagógico-educacionais em hospitais. 0 número de professores atuantes varia de um mínimo de um até um máximo de nove professores em cada classe hospitalar. A média de professores em cada hospital que abriga uma classe hospitalar foi de três professores por hospital.

A tabela seguinte mostra, por região, o número de professores atuando em classes hospitalares, assim como o quantitativo destes professores que responderam à segunda etapa da pesquisa.

Professores em Classes Hospitalares, por Região

\begin{tabular}{||l|c|c||}
\hline \multicolumn{1}{|c|}{ Região } & $\begin{array}{c}\text { Professo- } \\
\text { res em CH }\end{array}$ & $\begin{array}{c}\text { Prof. que } \\
\text { responde- } \\
\text { ram à 2a } \\
\text { etapa }\end{array}$ \\
\hline Norte & 10 & 09 \\
\hline Nordeste & - & 01 \\
\hline Centro-0este & 18 & 06 \\
\hline Sudeste & 29 & 22 \\
\hline Sul & 23 & 21 \\
\hline Total & 80 & $59(\mathbf{7 4} \%)$ \\
\hline
\end{tabular}

Responderam à segunda etapa da pesquisa $74 \%$ do total geral de professores que atuam em classes hospitalares. Deste total de 59 professores, 44\% possuem formação superior $(n=26)$, seguindo-se aqueles que concluíram formação básica de magistério, equivalente ao nível médio $(n=15,25 \%)$. Onze professores (19\%) possuem curso de pós-graduação (aperfeiçoamento, especialização, mestrado ou doutorado). Os demais professores $(n=7,12 \%)$ não especificaram seu grau de escolaridade. A formação universitária ou de pós-graduação comporta 63\% $(n=37)$ dos professores nas classes hospitalares, representando uma elevada qualificação formal entre aqueles que exercem a docência nestas classes.

A tabela seguinte sintetiza a formação dos professores de classe hospitalar. 
Formação Acadêmica dos Professores

\begin{tabular}{||l|c|c|}
\hline \multicolumn{1}{|c|}{ Nível Educacional } & $\mathbf{N}^{0}$ Prof. & $\mathbf{\%}$ \\
\hline Educação de nível médio & 15 & 19 \\
\hline Educação de nível superior & 26 & 32 \\
\hline Pós-graduação & 11 & 14 \\
\hline Sem Informação & 28 & 35 \\
\hline Total & $\mathbf{8 0}$ & $\mathbf{1 0 0}$ \\
\hline
\end{tabular}

No que se refere ao tempo de atuação no magistério entre os professores que responderam à segunda etapa da pesquisa, 22\% dos professores $(n=13)$ têm entre seis e dez anos de atuação profissional e outros 20\% $(n=12)$ têm mais de 20 anos de exercício na área educacional. Especificamente relacionado à atuação em classes hospitalares, a grande maioria dos professores $(68 \%, n=40)$ atua nessas classes há menos de cinco anos. Quinze por cento dos professores $(n=9)$ já atuam em classes hospitalares entre seis e dez anos, e apenas sete dos professores (12\%) estão há mais de dez anos trabalhando nas classes hospitalares. Três professores (5\%) deixaram de informar seu tempo de atuação nessa modalidade de atendimento pedagógico-educacional.

A faixa etária da maioria do corpo docente de classes hospitalares oscila entre 30 e 39 anos $(34 \%, n=20)$ com outros 32\% dos professores $(n=19)$ entre 40 e 49 anos. Cinco professores têm acima de 50 anos de idade $(8,5 \%)$. Oito dos professores $(13,6 \%)$ têm menos de 30 anos de idade. Sete professores $(11,9 \%)$ não informaram idade.

As atividades pedagógico-educacionais relacionadas às séries iniciais da educação básica (da alfabetização à $4^{\text {a }}$ série) envolvem 66\% dos professores que atuam em classes hospitalares. Os docentes das classes hospitalares dão prioridade ao atendimento relativo ao período de escolaridade obrigatória, o que denota uma clara preocupação com a manutenção dos vínculos escolares da aprendizagem no atendimento destas classes.
Um percentual de $18 \%$ dos professores atua em atividades pedagógico-educacionais relacionadas à educação infantil (crianças até seis anos de idade). Os demais 16\% atuam em atividades pedagógico-educacionais da educação básica à educação de nível médio. A tabela abaixo resume os dados deste parágrafo.

Atendimento em Classes Hospitalares

\begin{tabular}{||l|c||}
\hline Grupos atendidos em C. H. & \% \\
\hline Alfabetização à $4^{\mathrm{a}}$ série & 66 (Prioritário) \\
\hline Educação Infantil & 18 \\
\hline Educação básica e nível médio & 16 \\
\hline
\end{tabular}

\section{Perfil discente}

A grande maioria da clientela atendida em classes hospitalares tem idade abaixo de 15 anos (60\%). A idade máxima mencionada para atendimento foi de 18 anos. As crianças com menos de três anos de idade compreendem 14\% do quantitativo atendido.

0 número médio de crianças atendidas em cada classe hospitalar por mês é de 60 alunos, variando de um mínimo de dez a um máximo de 200 crianças, o que está diretamente ligado ao quantitativo de professores em cada classe.

A tabela, a seguir, mostra a média mensal de alunos atendidos pelas classes hospitalares, de acordo com a região do país em que estas se situam.

Alunos Atendidos Mensalmente, por Região

\begin{tabular}{||l|c|c||}
\hline \multicolumn{1}{|c|}{ Região } & $\begin{array}{c}\text { Alunos } \\
\text { atendidos/mês }\end{array}$ & $\begin{array}{c}\mathbf{N}^{\circ} \mathbf{~ d e} \\
\mathbf{C . H .}\end{array}$ \\
\hline Norte & 235 & 02 \\
\hline Nordeste & - & 03 \\
\hline Centro-0este & 305 & 09 \\
\hline Sudeste & 439 & 10 \\
\hline Sul & 469 & 06 \\
\hline Total & $\mathbf{1 . 4 4 8 *}$ & $\mathbf{3 0}$ \\
\hline
\end{tabular}

*Quatro classes hospitalares não informaram o quantitativo de alunos/mês e 14 não responderam à $2^{a}$ etapa da pesquisa. 
Em geral, um percentual de 73\% das crianças atende de três a quatro horas diárias de aula e chegam a ter uma média de dez aulas (61\% das crianças) durante seu período de internação. Um período de internação pode compreender até dez dias para 44\% das crianças e igual percentual de crianças (44\%) fica hospitalizado de $20(25 \%)$ a mais que $30(19 \%)$ dias.

No que se refere à problemática de saúde que causou a internação da criança que participa das atividades pedagógico-educacionais da classe hospitalar, o diagnóstico mais freqüente foi a desnutrição (22\%), principalmente para a faixa etária abaixo dos cinco anos de idade. A outra causa mais freqüente foi a pneumonia $(12 \%)$ que pode, ou não, estar associada à desnutrição ou outra enfermidade (por exemplo, a SIDA, com 5\% de ocorrência).'

Problemas oncológicos acometem 12\%; e renais, $8 \%$ da clientela atendida pelas classes hospitalares. Os demais $41 \%$ dos diagnósticos referem-se a diversas outras problemáticas de saúde (por exemplo: problemas ortopédicos, doenças cardíacas, congênitas etc.), algumas crônicas ou letais e que requerem internações periódicas (por exemplo: fibrose cística). Apenas $24 \%$ das classes hospitalares recebem esse tipo de clientela.

Uma vez que as classes hospitalares em geral desenvolvem suas atividades pedagógico-educacionais com grupos de crianças de idade variadas, não nos foi possível definir os diagnóticos por grupo etário.

\section{Políticas e diretrizes do trabalho de classe hospitalar}

Em geral, as classes hospitalares decorrem de convênio entre as Secretarias Estaduais ou Municipais de Educação e de Saúde dos estados (60\%). Entretanto, há, também, parcerias entre as Secretarias de Educação e entidades particulares e/ou filantrópicas e Universidades (40\%).

Assim, suporte pedagógico e material, além do corpo docente, são encargos da área de Educação. Cinqüenta por cento dos professores em exercício nas classes hospitalares recebem algum tipo de orientação ou treinamento pedagógico específico voltado para sua atuação nessas classes.

A área de Saúde tem a responsabilidade de ceder espaço físico aos professores para a atuação pedagógico-educacional no ambiente hospitalar. Reuniões entre a equipe de saúde e os professores ocorrem em 70\% dos hospitais que têm classes hospitalares. Em $40 \%$ destes hospitais, os professores também dispõem de suporte psicológico oferecido pela Secretaria de Saúde.

Do ponto de vista administrativo, e considerando apenas as classes hospitalares que participaram da segunda etapa da pesquisa $(n=16)$, sete delas (44\%) funcionam como uma escola regular ou como um anexo de uma escola regular. Oito classes hospitalares (50\%) contam com profissional exclusivo para coordenar o trabalho delas. Nas demais classes, é um dos professores regentes da classe que a representa e responde administrativamente por ela.

Quarenta por cento das classes hospitalares seguem a política e diretrizes da Educação Especial, as restantes seguem as diretrizes contidas na LDB ou em legislação educacional e/ou sanitária de seu estado ou município. Duas classes hospitalares informaram dispor de legislação específica formulada para esta modalidade de atendimento.

\section{Acompanhamento da escolaridade do aluno na}

classe hospitalar

Em sua prática pedagógico-educacional diária, as classes hospitalares visam dar continuidade ao ensino dos conteúdos da es-

1. É possível insinuar, com base nos prováveis diagnósticos médicos que concernem à presença do evento desnutrição na internação pediátrica, que este motivo de internação envolva outros eventos fisiopatológicos (a desidratação e as gastroenterites, por exemplo), da mesma forma que é possível insinuar a elisão dos diagnósticos de broncopneumonia, bronquiolite ou bronquite sob a designação pneumonia, entre os motivos de internação das crianças alunas das classes hospitalares. 
cola de origem da criança ou adolescente, e/ou operam com conteúdos programáticos próprios à faixa etária das crianças e jovens hospitalizados, o que os leva a sanar dificuldades de aprendizagem e/ou a oportunidade de aquisição de novos conteúdos intelectivos.

As classes hospitalares também atuam em intervenção pedagógico-educacional não propriamente relacionada à experiência escolar, mas às necessidades do desenvolvimento psíquico e cognitivo da criança ou adolescente (Fonseca, 1996; Fonseca \& Ceccim, 1999).

Cinqüenta por cento das classes hospitalares mantêm contato com a escola regular de origem da criança ou adolescente hospitalizado.

A possibilidade de atendimento em classes hospitalares serve à manutenção das aprendizagens escolares, ao retorno e à reintegração da criança ou jovem ao seu grupo escolar, e como acesso à escola regular, uma vez que algumas das crianças hospitalizadas em idade de freqüência escolar não estão formalmente matriculadas na rede de ensino.

Quando a ausência da criança na escola decorre de sua história de adoecimento e tratamento hospitalar, a freqüência à classe hospitalar incentiva a criança e a família a buscarem a escola regular após a alta hospitalar.

\section{Discussão e conclusões}

Até esta pesquisa, não havia sido organizado nenhum documento com informações sobre o atendimento pedagógico-educacional no ambiente hospitalar, no Brasil. Foram detectadas 30 classes hospitalares no território brasileiro. As regiões Centro-Oeste, Sudeste e Sul apresentam maior número de classes hospitalares, se considerado o total de estados em cada uma dessas regiões. Estas três regiões somam 11 Estados e 25 classes hospitalares. As regiões Norte e Nordeste somam 16 Estados e 5 classes hospitalares. Do universo de 27 unidades da federação, apenas 11 possuem classes hospitalares. Dentre as 30 classes, $44 \%$ se distinguem do serviço de recreação e apenas dez possuem salas de aula.

A educação em hospital é um direito de toda criança ou adolescente hospitalizado. Os resultados aqui apresentados demonstram que, na prática, nem todas as crianças estão tendo este direito respeitado ou atendido, uma vez que os dados evidenciam um número pequeno de hospitais com classes hospitalares. Faz-se necessário considerar, seriamente, esta questão, uma vez que a literatura aponta para o importante papel do professor no desenvolvimento, nas aprendizagens e no resgate da saúde pela criança ou adolescente hospitalizado.

Conforme destaca Wiles, "a função do professor de classe hospitalar não é apenas a de manter as crianças ocupadas" (1987, p.640), ele é capaz de incentivar o crescimento e desenvolvimento somatopsíquico, intelectivo e sócio-interativo. Uma vez que a criança não tem seu crescimento e desenvolvimento interrompidos por estar hospitalizada, a presença do professor que conhece as necessidades curriculares desta criança torna-se um catalisador que, ao interagir com a criança, proporciona-lhe condições para a aprendizagem. Isto aproxima a criança dos padrões cotidianos da vida. Corroborando o exposto anteriormente, Ceccim e colaboradores acrescentam que é também do professor de classe hospitalar "a tarefa de afirmar a vida, e sua melhor qualidade, junto com essas crianças, ajudando-as a reagir, interagindo para que o mundo de fora continue dentro do hospital e as acolha com um projeto de saúde" (1997, p.80).

A oferta de atividades recreativas e/ou lúdicas no ambiente de internação hospitalar é crucial ao enfrentamento do adoecimento e à aceitação positiva do tratamento, mas não substitui a necessidade de atenção pedagógico-educacional, pois seu potencial de intervenção é mais específico, mais individualizado e volta-se às construções cognitivas e à 
construção do desenvolvimento psíquico (Fonseca, 1998; Fonseca \& Ceccim, 1999). A classe hospitalar pode partir de programas lúdicos voltados à infância, mas sua ênfase recai em programas sócio-interativos de desenvolvimento e educação da criança e do adolescente hospitalizados, vinculando-se aos sistemas educacionais como modalidade de ensino (Educação Especial) ou aos sistemas de saúde como modalidade de atenção integral (Atendimento Pedagógico-Educacional Hospitalar).

A grande maioria dos professores tem menos de cinco anos de atuação nessa modalidade de atendimento. Pode-se propor que tal fato se deva ao recente crescimento da oferta/procura nessa área, uma vez que tais professores já atuavam em escolas regulares por tempo significativo. Embora não diretamente relacionado ao escopo deste estudo, é importante ressaltar uma possível relação entre o significativo aumento do número de classes hospitalares implantadas a partir da década de 1980 e as mudanças políticas e os avanços científicos neste mesmo período nas áreas pediátrica, pedagógica, de educação básica e de saúde coletiva.

No que se refere à formação acadêmica dos professores que atuam em classes hospitalares, devido ao tipo de instrumento utilizado para a coleta de dados, não foi possível verificar suas áreas específicas de habilitação, o que sugere a formulação de nova etapa de investigação neste aspecto. Apesar disso, parece relevante destacar que o número de professores nas classes hospitalares é expressivamente composto por profissionais com nível superior (46\% do total de professores ou 63\% entre os que responderam à $2^{\text {a }}$ etapa da pesquisa) e $30 \%$ do total de professores possuem pós-graduação. 0 desconhecimento da formação docente de 35\% do total de professores nas classes hospitalares não nos sugere inversão na tendência de que a atuação nas classes hospitalares seja majoritária aos profissionais com educação superior. Mesmo que o atendi- mento pedagógico-educacional em hospitais não requeira formação específica, essa atividade requer profissionais com destreza e discernimento para atuar com planos e programas abertos, móveis, mutantes, constantemente reorientados pela situação especial e individual de cada criança sob atendimento (Fonseca \& Ceccim, 1999). A maior formação do professor o capacita para lidar com referências subjetivas das crianças e para a busca de literatura de alto nível intelectual (única disponível, muitas vezes em língua estrangeira).

As classes hospitalares são, em geral, resultado de convênio entre as Secretarias de Educação e de Saúde. Parece relevante ressaltar que, cabendo aos hospitais basicamente ceder espaço para a instalação das classes hospitalares, este atendimento pedagógico-educacional tende a ocorrer nas enfermarias, o que denota não haver, por parte dos hospitais, o cuidado com o espaço a ser utilizado por esta modalidade de atendimento. Devemos, então, considerar a necessidade de clarificar aos hospitais o trabalho realizado pelas classes hospitalares, a fim de que estas possam dispor de acomodações mais adequadas para o exercício de suas atividades. Faz-se necessário transpor barreiras e, por meio de esforços unificados, garantir a excelência dos serviços, sejam estes prestados por professores, pessoal da saúde ou quaisquer outros profissionais que atuam no ambiente hospitalar (Fonseca, 1995a), contribuindo, assim, para a qualificação da assistência prestada em hospitais.

A sistemática de atuação das classes hospitalares no Brasil é semelhante à que norteia esse tipo de trabalho em outros países. Por exemplo, em 0xford na Inglaterra, o atendimento pedagógico-educacional oferecido visa, assim como no Brasil, dar continuidade ao processo de desenvolvimento e ao processo de aprendizagem das crianças e jovens hospitalizados (Fassam, 1982). 
Uma vez que não se obtiveram informações detalhadas de todas as classes hospitalares mapeadas na $1^{\text {a }}$ etapa da pesquisa, eleva-se, agora, a necessidade de melhor conhecer essa realidade, pois conhecido o universo quantitativo, desconhecemos os princípios norteadores, as práticas levadas a efeito e as repercussões na educação e na saúde da criança e do adolescente. Em outras palavras, o atendimento de classe hospitalar precisa ser estudado e discutido mais profundamente, dentro e fora de seu grupo profissional imediato, para que o papel e propostas do professor diante das crianças com diversas idades, habilidades e necessidades sejam mais efetivamente implementados (enfoque interdisciplinar).

Os resultados do presente estudo, assim como a remessa do seu resumo para os profissionais participantes deste, reforçam a possibilidade de reflexão crítica sobre a realidade com que tais profissionais se defrontam e os procedimentos que utilizam em sua prática diária. Também contribuem para a ampliação desta discussão com as equipes de saúde dos hospitais que dispõem desta modalidade de atendimento. Paralelamente a esta discussão, outros projetos urgem para implementação, e, conforme o exposto nos parágrafos anteriores, poderão considerar os aspectos abaixo sintetizados:
- obter informações pormenorizadas das classes existentes, incluindo visitas a tais espaços físicos e entrevistas com professores, profissionais de saúde, crianças e familiares (e detalhes sobre questões administrativas, tais como recebimento de adicional de insalubridade e de regência de classe pelos professores das classes hospitalares);

- acessar pontos de vista dos pais e das crianças sobre classe hospitalar, assim como da equipe de saúde do hospital;

- divulgar na sociedade e informar famílias sobre a existência desta modalidade de atendimento e que a criança tem direito de usufruir deste serviço;

- analisar a contribuição desta modalidade de atendimento no desempenho escolar da criança, após a alta e retorno à sua escola de origem;

- analisar a contribuição deste atendimento na resposta clínica da criança ou adolescente ao tratamento hospitalar.

Em termos gerais, os resultados desta pesquisa contribuem para a discussão sobre as questões específicas desta modalidade de atendimento, levando-nos à elaboração futura de uma política voltada para os direitos pedagógico-educacionais das crianças e jovens hospitalizados.

\section{Referências bibliográficas}

BRASIL. Ministério da Educação e do Desporto. Secretaria de Educação Especial. Política Nacional de Educação Especial. Brasília: MEC/SEESP, 1994. 66p, livro1.

CECCIM, Ricardo B. et al. Escuta pedagógica à criança hospitalizada. In: CECCIM, Ricardo B.; CARVALHO, Paulo R. A (Orgs.) Criança hospitalizada: atenção integral como escuta à vida. Porto Alegre: Editora da Universidade/UFRGS, 1997. p.76-84.

CONSELHO NACIONAL DOS DIREITOS DA CRIANCA E DO ADOLESCENTE. Resolução n 41, de 13 de outubro de 1995. Direitos da criança e do adolescente hospitalizados. Brasília: Imprensa Oficial, 1995.

FASSAM, Meg. Education for children in hospital. London: The National Association for Children in Hospital, 1982. 28p.

FONSECA, Eneida S. Classe hospitalar: uma modalidade válida da educação especial no atendimento precoce? In: SEMINÁRIO BRASILEIRO DE PESQUISA EM EDUCACAO ESPECIAL, 5. Niterói, 1996. Anais. Rio de Janeiro: UFF, 1996. p.37. 
Programa de Pesquisas para o Desenvolvimento de Estratégias Ligadas aos Direitos e Necessidades Pedagógico-Educacionais de Crianças e Jovens Hospitalizados. Rio de Janeiro, 1995. 43p. [mimeografado]

Young children's distress during radiological examinations. Londres, 1995. 322p. Tese (PhD) - Institute of Education/University of London and the Department of Pediatric Radiology/The Great Ormond Street Children's Hospital.

.; CECCIM, Ricardo B. Atendimento pedagógico-educacional hospitalar: promoção do desenvolvimento psíquico e cognitivo da criança hospitalizada. Temas sobre Desenvolvimento, São Paulo, v.7, n.42, p.24-36, jan./fev. 1999.

WILES, Paddy M. The schoolteacher on the hospital ward. Journal of Advanced Nursing, Londres, n.12, p.631-640, 1987.

Recebido em 12 maio 1999

Aprovado em 05 out. 1999 